\title{
Mediterranean diet adherence and cardiovascular risk factors in a South Asian population: A cross-sectional study
}

\author{
I. Saeid, L. O'Connor and T.J. Butler \\ Department of Health Professions, Manchester Metropolitan University, Manchester, M15 6BG
}

South Asian populations are at higher risk of cardiovascular disease (CVD) in comparison to other ethnicities, showing an earlier onset of coronary heart disease and stroke ${ }^{(1,2)}$ with genetic predisposition to higher circulating lipoprotein a and triacylglycerol as explanations ${ }^{(3)}$. Diet quality is also associated with CVD risk. The Mediterranean diet (MD) has been encouraged as a cardioprotective eating pattern ${ }^{(4)}$ and has well-established links with reduced rates of CVD. The aim of the present study was to examine the applicability of a MD score (MDS) tool to a South Asian population and assess prevalence of lifestyle risk factors for CVD.

Participants were sampled from local community settings in central Manchester, UK. Those meeting entry criteria of South Asian ethnicity and current absence of overt CVD were assessed for adherence to the MD using a validated 55-point questionnaire ${ }^{(5)}$. Overall dietary intake was assessed using a 3-day food diary. Self-reported weight and height were recorded, and BMI calculated. Smoking status and physical activity level were also examined. All procedures were approved by the research ethics committee at Manchester Metropolitan University.

\begin{tabular}{|c|c|c|c|c|c|c|c|}
\hline \multirow[b]{2}{*}{ Group } & \multicolumn{2}{|c|}{ Weight (kg) } & \multicolumn{2}{|c|}{ BMI $\left(\mathrm{kg} / \mathrm{m}^{2}\right)$} & \multirow{2}{*}{$\begin{array}{l}\text { Current smoker } \\
\text { Frequency }(\%)\end{array}$} & \multicolumn{2}{|l|}{ MDS } \\
\hline & Mean & $\mathrm{SD}$ & Mean & $\overline{\mathrm{SD}}$ & & Mean & $\mathrm{SD}$ \\
\hline Total (n 50) & 64.7 & $15 \cdot 7$ & $20 \cdot 5$ & 4.7 & $7(14)$ & $26 \cdot 3$ & $6 \cdot 3$ \\
\hline Men ( $n$ 20) & $78 \cdot 6$ & $15 \cdot 2$ & $27 \cdot 5$ & $4 \cdot 5$ & $7(35)$ & $24 \cdot 5$ & $7 \cdot 1$ \\
\hline Women (n 30) & $60 \cdot 0^{*}$ & $11 \cdot 2$ & $23 \cdot 5 * *$ & $4 \cdot 3$ & $0(0)^{*}$ & $27 \cdot 6$ & $5 \cdot 4$ \\
\hline
\end{tabular}

MDS, Mediterranean diet score. $* P<0.001$ and $* * P=0.006$ vs. men.

${ }^{\ddagger}<0.001$ vs. men

Of the 108 screened individuals, 90 met the inclusion criteria. 50 people attended clinics for anthropometric and dietary assessment and were included in analyses. Men had higher weight, BMI and a greater number were current smokers in comparison to women $(P<0.001, P=0.006$, and $P<0.001$, respectively). MDS was $26.3 \pm 6.3$ for the total group. No significance differences were observed between men and women in MDS. A greater number of servings of fruit, vegetables, and legumes were found in the highest tertile of MDS vs. the lowest, and fewer servings of full-fat dairy, red meat and meat products, and poultry $(P<0.001)$. The percentage of women in tertile 2 and 3 of the MDS was significantly $(P=0.02)$ greater than men, whereas men contributed a greater percentage of the lowest tertile. No differences in energy and protein intake were observed between men and women (1934 $\pm 544 \mathrm{kcal}$ vs. $1663 \pm 469 \mathrm{kcal}, P=0 \cdot 06 ; 78 \cdot 1 \pm 24.5 \mathrm{~g} / \mathrm{d}$ vs. $65 \cdot 5 \pm 22 \cdot 9, P=0 \cdot 06$, respectively).

In conclusion, these findings suggest that in a South Asian population a higher intake of fruit, vegetables, and legumes, and lower intake of meat and dairy are indicators of greater adherence to a cardioprotective diet. South Asian men display a greater number of $\mathrm{CV}$ risk factors such as greater weight and smoking prevalence. This can be of importance when interpreting future studies in this area and in the design of CVD prevention strategies.

1. Hussain SM, Oldenburg B, Wang Y et al. (2013). Int J Vasc Med 2013 786801-786810.

2. Forouhi NG, Sattar N, Tillin T et al. (2006). Diabetologia 49, 2580-258.

3. Bilen O, Kamal A, Virani SS. (2016) World J Cardiol 8, 247-257.

4. Gerber M \& Hoffman R (2015) Br J Nutr 113, S14-S10.

5. Panagiotakos DB, Pitsavos C, Arvaniti F et al. (2007). Preventive medicine 44, 335-340. 